\title{
Efeitos de um programa de exercícios em grupo sobre a força de preensão manual em idosas com baixa massa óssea
}

\author{
Effects of a group exercise program on handgrip \\ strength of elderly women with low bone mass
}

1 Departamento de

Fisioterapia, Universidade Federal de Pernambuco (UFPE), Recife, PE, Brasil
Correspondência para: Márcia Alessandra Carneiro Pedrosa Rua Agricolândia, 171, ap. 303 Cidade Universitária 50740-470 - Recife, PE, Brasil macpedrosa@yahoo.com.br

Recebido em 18/Abr/2012 Aceito em 21/Jun/2012

\begin{abstract}
Eduarda Lubambo Costa' ', Paulo Sérgio de Castro Bastos Filho', Mariana de Sousa Moura' ', Tiago Siqueira de Sousa', Andréa Lemos', Márcia Alessandra Carneiro Pedrosa'
\end{abstract}

\section{RESUMO}

Objetivos: Avaliar os efeitos de um programa de exercícios sobre a força de preensão manual (FPM) em idosas com baixa massa óssea. Sujeitos e métodos: Trinta e seis idosas foram randomizadas em: grupo intervenção - $\mathrm{GI}(\mathrm{n}=18)$ e grupo controle - $\mathrm{GC}(\mathrm{n}=18)$, avaliadas pelo teste de FPM, utilizando-se um dinamômetro de mão. O Gl realizou exercícios de força muscular, equilíbrio e mobilidade funcional. O GC realizou alongamentos. Ambos os grupos realizaram duas sessões semanais de 50 minutos, durante quatro meses. Resultados: O GI apresentou aumento da FPM em 31,38\% ( $p=0,00)$. O GC não mostrou diferença. Houve correlações entre a FPM e a densidade mineral óssea (DMO) do colo do fêmur $(r=0,582, p=0,003)$ e do fêmur total $(r=0,485, p=0,01)$. Conclusão: 0 s resultados sugerem uma tendência ao incremento da FPM de idosas e mostraram correlações significantes entre a FPM e a DMO. Arq Bras Endocrinol Metab. 2012;56(5):313-8

Descritores

Densidade óssea; exercício; força muscular; osteoporose; saúde do idoso; terapia por exercício

\section{ABSTRACT}

Objectives: To evaluate the effects of an exercise program on handgrip strength (HS) in elderly women with low bone mass. Subjects and methods: Thirty-six elderly women were randomized to the intervention group - $\mathrm{Gl}(\mathrm{n}=18)$ and control group - $\mathrm{GC}(\mathrm{n}=18)$. Participants were evaluated by means of a HS test, using a handgrip dynamometer. Gl performed strength, balance and functional mobility exercises, and GC performed stretching exercises. Both groups had sessions twice a week for 50 minutes, during 4 months. Results: Gl had a significant increase in handgrip strength equal to $31.38 \%$. No differences were observed in GC. There were correlations between HS test and bone mineral density (BMD) of the femoral neck $(r=0.582, p=0.003)$ and total femur $(r=0.485, p=0.01)$. Conclusion: Results indicate a propensity to increase elderly HS, and showed significant correlations between HS and BMD. Arq Bras Endocrinol Metab. 2012;56(5):313-8

\section{Keywords}

Bone density; exercises; muscle strength; osteoporosis; elderly health; exercise therapy

\section{INTRODUÇÃO}

$\mathrm{U}$ ma das alterações decorrentes do processo de envelhecimento é a redução da massa óssea, que pode levar à osteoporose $(1,2)$, uma doença caracterizada pela deterioração da microarquitetura do tecido ósseo e aumento da fragilidade mecânica, tornando o osso predisposto a fraturas por traumas mínimos (3).

Outra modificação característica do envelhecimento é a sarcopenia $(4,5)$, que leva à fraqueza muscular e contribui para a diminuição da capacidade funcional do 
idoso $(4,6)$. Dentre as várias maneiras de avaliar a força muscular, a mensuração da força de preensão manual (FPM) por meio do dinamômetro de mão é entendida como indicadora geral da força e potência musculares (7). Geraldes e cols. (8) afirmam que a FPM é o melhor indicador da capacidade do sistema musculoesquelético e do desempenho funcional de idosos institucionalizados.

A fraqueza muscular juntamente com o comprometimento do equilíbrio e a redução da velocidade de reação são, pelo menos em parte, responsáveis pelo aumento do número de quedas entre os idosos (9-11).

Dentre as estratégias para prevenção de quedas, manutenção da força muscular e da densidade mineral óssea, o exercício físico destaca-se como uma das mais importantes (12-16). Este estudo tem por objetivo avaliar os efeitos de um programa em grupo de exercícios resistidos e de equilíbrio sobre a força de preensão manual palmar em idosas com baixa massa óssea. Como desfecho secundário, pretende-se avaliar as correlações entre a força de preensão manual e a densidade mineral óssea.

\section{MÉTODOS}

Trata-se de um estudo piloto de um ensaio clínico randomizado e controlado, no qual foram avaliadas mulheres idosas atendidas no ambulatório de Reumatologia Clínica do Hospital das Clínicas (HC) da Universidade Federal de Pernambuco (UFPE), no período de setembro a novembro de 2009. As participantes foram divididas em dois grupos - grupo intervenção (GI) e grupo controle (GC) - mediante uma randomização simples realizada pelo computador por meio do programa SPSS (Statistical Package for the Social Sciences) versão 16.0.

Foram considerados como critérios de inclusão: mulheres idosas na faixa etária de 60 a 88 anos, não institucionalizadas, capazes de entender e de responder aos comandos utilizados durante avaliação (baseado no Miniexame do Estado Mental) (17) - e com deambulação presente sem assistência ou supervisão de outros; com diagnóstico de osteopenia ou osteoporose definidos respectivamente, conforme critério da OMS (3), por um T-score de - 1 a -2,4 desvio-padrão (DP) e por um T-score -2,5 DP abaixo da média para mulheres brancas com idade entre 20 e 30 anos a partir do exame de densitometria óssea por raios X de dupla energia (DXA), a nível da coluna lombar Ll-L4, colo femoral ou fềmur total.

Como critérios de exclusão, consideraram-se: prática de atividade física regular duas vezes por semana ou mais; diagnóstico médico de vestibulopatias, doenças neuromusculares e hipertensão arterial não controlada; uso de benzodiazepínicos e neurolépticos; e incapacidade de realizar os testes de preensão manual palmar.

Foram incluídas 36 pacientes, sendo que o grupo intervenção $(\mathrm{n}=18)$ realizou exercícios resistidos e exercícios de equilíbrio e o grupo controle $(\mathrm{n}=18)$ realizou exercícios de alongamento muscular e recebeu palestras informativas sobre osteoporose, quedas e outros temas relacionados à saúde do idoso.

O estudo foi previamente aprovado pelo Comitê de Ética em Pesquisa do Centro de Ciências da Saúde da UFPE, sob o número de protocolo: 0359.0.172.00008. Os participantes concordaram em participar do estudo após assinatura do termo de consentimento livre e esclarecido.

\section{Avaliação da força de preensão manual}

O teste de força de preensão manual foi realizado com o dinamômetro de mão (Saehan Corporation ${ }^{\circledR}$, modelo SH5001), com o braço em posição neutra, cotovelo flexionado a $90^{\circ}$ e antebraço entre a pronação e supinação. O paciente foi instruído a apertar o instrumento com o máximo de força, utilizando a mão dominante. Durante a realização do teste foi utilizado pelo examinador o comando verbal "mais força" para incentivar o paciente a realmente realizar o teste com sua força máxima. Três medidas foram realizadas e a média entre elas foi calculada $(7,18,19)$.

\section{Intervenção}

A intervenção cinesioterapêutica foi realizada por cinco pesquisadores previamente treinados para aplicar o programa de exercícios e para monitorizar os voluntários. As sessões de ambos os grupos foram realizadas no setor de Fisioterapia do HC-UFPE, duas vezes por semana, com duração de 50 minutos cada sessão, por um período de quatro meses, totalizando 30 atendimentos para cada grupo. Os dois grupos realizaram as atividades em horários distintos, para não tomarem conhecimento da conduta realizada pelo outro grupo.

O programa de exercícios do grupo intervenção foi baseado nas recomendações da International Osteoporosis Foundation - IOF (20). A sessão do grupo intervenção foi composta de dez minutos de aquecimento, trinta minutos de treinamento e dez minutos de desaquecimento e relaxamento. O treinamento foi constituído de treino de força muscular e treino de equilíbrio e 
mobilidade funcional, de modo que essas modalidades de treinamento foram realizadas em dias distintos e em sessões alternadas, no qual em cada sessão foi realizada apenas uma das formas de treinamento.

Durante o aquecimento, foram realizados exercícios lúdicos com associação de movimentos livres de membros superiores ao deslocamento anterior, posterior e lateral do corpo. Em seguida, foram realizados alongamentos dos músculos: trapézio (fibras superiores), paravertebrais toracolombares, abdominais, quadríceps, adutores do quadril, isquiotibiais, gastrocnêmios e tibial anterior. O alongamento de cada músculo foi realizado uma vez por 15 segundos, com o participante em pé.

No treinamento de força muscular, foram realizados exercícios resistidos para os flexores e extensores do tronco (músculos abdominais, eretores espinhais e paravertebrais toracolombares); flexores, extensores, abdutores e adutores de quadril; flexores e extensores de joelho e flexores plantares do tornozelo realizados em cadeia cinética aberta. Foram feitos miniagachamentos $\left(0-45^{\circ}\right)$ em cadeia cinética fechada (21). Os exercícios foram realizados em três séries de dez repetições, utilizando-se inicialmente resistência gravitacional e posteriormente resistência elástica e pesos com velcro, com base na capacidade do indivíduo.

No treinamento de equilíbrio, foram praticados exercícios de equilíbrio estático e equilíbrio dinâmico associados ao treinamento de mobilidade funcional. Foram realizados exercícios em posição ortostática com apoio bipodal (posição de Romberg - um pé ao lado do outro) e com base de apoio reduzida (posição de Romberg sensibilizada - um pé à frente do outro), com olhos abertos e com olhos fechados. Exercícios de flexão plantar e exercícios de equilíbrio sobre tábua de propriocepção tibiotársica também foram realizados. Os exercícios de equilíbrio unipodal foram feitos em três séries de dez repetições de flexão do joelho; flexão, extensão e abdução do quadril, e inclinação do tronco para frente com olhos abertos e fechados e equilíbrio sobre o balancinho. Após a $15^{\mathrm{a}}$ sessão, houve incremento na dificuldade das atividades de modo que a manutenção das posturas passou a ser de até 15 segundos, as atividades de equilíbrio unipodal foram realizadas com olhos fechados e os exercícios passaram a também ser executados sobre colchões de diferentes texturas.

Atividades lúdicas com bolas, bexigas e bastões que desafiavam a coordenação e estimulavam um rápido tempo de reação também foram realizadas no treina- mento do equilíbrio dinâmico e mobilidade funcional. Em seguida, realizava-se um circuito com seis pontos, sendo eles: treino de marcha sobre o solo com variações no tamanho do passo e movimentos cruzados de membros superiores e inferiores; treino de marcha com obstáculos; treino de marcha sobre colchões de diferentes texturas; treino de marcha na ponta dos pés; meio passo anterior na cama elástica; e atividades de subida e descida em degraus. Cada um desses exercícios era realizado por 1 minuto por cada participante.

No desaquecimento e relaxamento, foram feitas caminhadas leves por 3 minutos, em seguida foram realizados alongamentos dos mesmos grupos musculares utilizados no aquecimento, mas foram realizados com o participante deitado. Para concluir a sessão, foram realizados, com o paciente sentado, exercícios respiratórios de inspiração profunda com padrão diafragmático, em três séries de cinco repetições.

Nas sessões do grupo controle, foram realizados exercícios de alongamentos dos músculos: trapézio, escalenos, esternocleidomastóideo, peitoral maior e menor, romboides, deltoide, bíceps braquial, tríceps braquial, flexores e extensores do punho, paravertebrais, abdominais, quadríceps, adutores do quadril, isquiostibiais, gastrocnêmios e tibial anterior. $\mathrm{O}$ alongamento de cada músculo foi realizado uma vez por um minuto. Foram realizados exercícios ativo-livres de flexão, abdução, extensão, rotação interna e externa do ombro; flexão e extensão do cotovelo e do punho com bastão em uma série de dez repetições. Foram feitos exercícios de relaxamento com movimentos de flexão ou abdução do ombro associado à inspiração e de extensão ou adução do ombro associado à expiração e exercícios respiratórios de respiração profunda com padrão diafragmático em três séries de cinco repetições. Também foram realizadas palestras informativas sobre osteoporose, quedas e outros temas relacionados à saúde do idoso. O objetivo deste grupo foi controlar os possíveis efeitos confundidores decorrentes da interação social e mudanças no estilo de vida em função da participação no estudo. Durante o estudo, os participantes dos dois grupos não realizaram outro tipo de atividade física.

\section{Análise estatística}

A análise dos dados foi realizada com o software de estatística SPSS versão 16.0. Para a análise estatística dos resultados, foram utilizados testes paramétricos, pois não houve significância nos testes de normalidade de 
Kolmogorov-Smirnov e de Shapiro-Wilk, evidenciando uma distribuição normal dos dados. Portanto, para comparar os dois grupos (GI e GC), foi utilizado o teste $t$ de Student para amostras não pareadas, de modo que os dois grupos apresentaram-se homogêneos (Tabela 1).

Após a intervenção, os testes foram repetidos e os resultados obtidos foram analisados pelo mesmo software SPSS por meio dos testes $t$ de Student para amostras independentes e teste $t$ de Student para amostras pareadas.

As correlações entre a FPM e a DMO foram avaliadas mediante a correlação linear de Pearson. A significância estatística considerada foi $\mathrm{p}<0,05$.

\section{RESULTADOS}

Dos 300 pacientes atendidos no Ambulatório de Reumatologia Clínica, 36 idosas $(69,3 \pm 6,1$ anos) foram selecionadas e randomizadas em dois grupos: GI ( $\mathrm{n}=$ 18) - grupo intervenção e GC ( $\mathrm{n}=18)$ - grupo controle. Durante a intervenção, nove participantes (sendo cinco do grupo intervenção e quatro do grupo controle) abandonaram o estudo alegando motivos pessoais. Vinte e sete indivíduos participaram da reavaliação (Figura 1). A caracterização e a homogeneidade da amostra são demonstradas na tabela 1 .

As reavaliações foram realizadas após o término das 30 sessões de intervenção. A frequência média de participação foi 20,07 $( \pm 7,2)$ sessões para o GI e 18,56 ( \pm $7,7)$ sessões para o GC.

Tabela 1. Características basais clínicas, antropométricas e densidade mineral óssea dos grupos intervenção (G1) e controle (G2)

\begin{tabular}{lccc}
\hline Variáveis & G1 (n = 18) & G2 (n = 18) & p \\
\hline Idade (anos) & $69,39(6,76)$ & $69,39(5,73)$ & 1,000 \\
Altura (cm) & $149,30(6,22)$ & $150,30(4,51)$ & 0,630 \\
Peso (kg) & $57,33(11,88)$ & $59,16(12,72)$ & 0,699 \\
Índice de massa corporal & $25,69(4,93)$ & $26,19(5,52)$ & 0,804 \\
Miniexame do Estado Mental & $25,33(2,84)$ & $25,78(3,79)$ & 0,694 \\
(escore de 0-30) & & & \\
DM0 colo femoral (g/cm²) & $0,760(0,09)$ & $0,784(0,09)$ & 0,530 \\
DM0 trocânter do fêmur (g/cm²) & $0,691(0,08)$ & $0,676(0,09)$ & 0,673 \\
DM0 fêmur total (g/cm²) & $0,828(0,13)$ & $0,861(0,09)$ & 0,468 \\
DM0 coluna lombar (L1-L4) (g/cm²) & $0,820(0,13)$ & $0,902(0,16)$ & 0,168 \\
Teste de força de preensão & $10,25(3,7)$ & $11,92(4,2)$ & 0,240 \\
manual (kgf) & & & \\
\hline
\end{tabular}

Os dados são expressos como média (DP).

Teste $t$ de Student para amostras não pareadas; G1: grupo intervenção; G2: grupo controle; DP: desvio-padrão; DMO: densidade mineral óssea.
Após a aplicação do programa de exercícios, ao analisar o efeito da intervenção intragrupos, o GI apresentou melhora significante na FPM de $31,38 \%(\mathrm{p}=0,00)$, enquanto o GC não mostrou diferença (Figura 2). Na avaliação intergrupos, no entanto, não houve diferença entre eles.

Considerando-se as 36 pacientes avaliadas no início do estudo, as medidas da FPM correlacionaram-se positivamente com a densidade mineral óssea (DMO) do colo do fêmur $(\mathrm{r}=0,582, \mathrm{p}=0,003)$ e com a DMO do fềmur total $(\mathrm{r}=0,485, \mathrm{p}=0,01)$.

\section{DISCUSSÃO}

Os resultados encontrados mostram um aumento da força de preensão manual do grupo intervenção na análise intragrupos. Foram incluídas 36 idosas, neste estudo piloto, o que corresponde a $10,7 \%$ do cálculo amostral estimado. Portanto, os resultados observados poderão mostrar-se significantes, numa análise intergrupos, diante de um número amostral maior.

Muitos estudos mostram que os benefícios do exercício nos idosos são mais evidentes quando os programas de exercício são praticados por longos períodos (22-25). No nosso estudo, foi proposto um programa de exercícios por 17 semanas, o que representa um período curto em relação ao recomendado pela literatura (22), mas que mesmo assim apresentou resultados positivos na análise intragrupos.

Skelton e cols. (26) observaram melhora estatisticamente significante na força de preensão manual $(4 \%, \mathrm{p}$ $=0,05)$ no grupo de idosos que realizou um programa de exercícios resistidos por 12 semanas, três vezes por semana, em relação ao grupo controle que não realizou nenhum tipo de intervenção (26). Muitos estudos, como o de Skelton e cols. (26), avaliam a eficácia do exercício físico no incremento da força de preensão manual comparando o grupo intervenção com um grupo controle que não é submetido a nenhum tipo de intervenção (26). No nosso estudo, o grupo controle realizou exercícios de alongamento muscular, participando de atividades em grupo e consequentemente beneficiando-se do bem-estar que atividades em grupo promovem (27). Além disso, exercícios de alongamento aumentam a mobilidade dos tecidos moles e a amplitude de movimento articular, promovem relaxamento muscular e aliviam a dor (27), o que pode ter contribuído para a melhora da capacidade funcional dos idosos do grupo controle. 


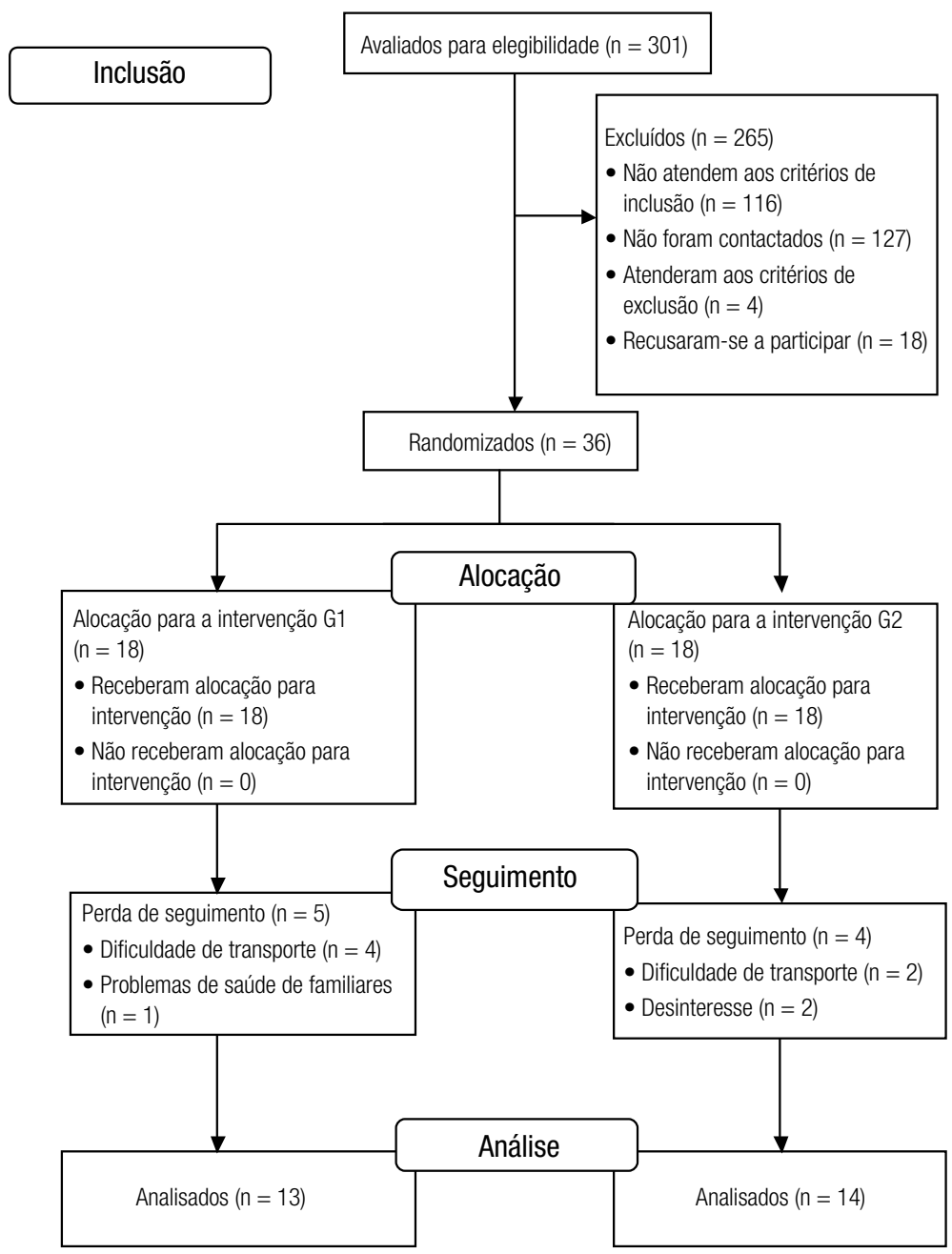

Figura 1. Fluxograma representando o número de participantes em cada etapa do estudo.

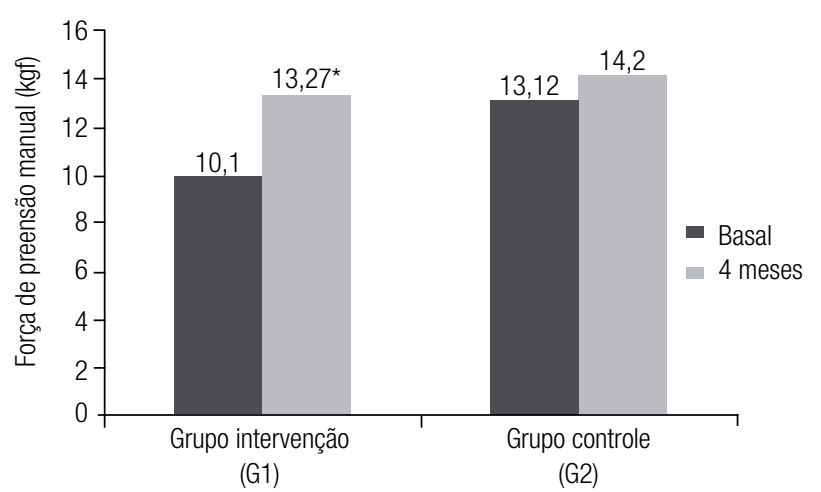

Figura 2. Progressão no desempenho do teste de Força de Preensão Manual (FPM) no Grupo Intervenção (G1) e no Grupo Controle (G2) na avaliação basal e após quatro meses de intervenção.

O presente estudo observou correlação direta entre a FPM e a DMO do colo femoral e do fêmur total, corroborando os achados encontrados na literatura, que mostram haver relação positiva entre força muscular e DMO de indivíduos idosos (12,28-31). Marin e cols. (30) avaliaram 117 mulheres pós-menopausa e observaram que, entre os testes de performance física, a FPM foi a que apresentou melhores correlações com a DMO da coluna lombar, colo do fêmur e corpo total. Esses autores também observaram correlações diretas e estatisticamente significantes entre a massa magra e DMO da coluna lombar $(\mathrm{r}=0,28)$, colo do fêmur $(\mathrm{r}$ $=0,32)$ e corpo total dos participantes $(r=0,49)$. Resultados semelhantes foram encontrados no estudo de Lima e cols. (31), os quais avaliaram 246 mulheres idosas, sendo classificadas como sarcopênicas aquelas cuja massa magra apendicular apresentava-se inferior a 5,45 $\mathrm{kg} / \mathrm{m}^{2}$. Neste estudo, a força do músculo extensor do joelho (mensurada por meio do pico de torque isocinético) e a massa magra de corpo total foram significativa e positivamente correlacionadas com a DMO de corpo 
total, coluna lombar, colo femoral, trocânter e triângulo de Ward. Por outro lado, as mulheres sarcopênicas apresentavam valores significativamente menores de DMO de corpo total e de trocânter, quando comparadas às não sarcopênicas. Esses estudos demonstram a importância da força muscular em idosas fisicamente ativas para manutenção da saúde óssea (30-31).

Os resultados encontrados sugerem uma tendência a um incremento da força de preensão manual de idosas com baixa massa óssea e comprovam correlações diretas entre a força de preensão manual e a densidade mineral óssea. No entanto, um período de tempo de intervenção mais longo e um número amostral maior poderiam confirmar os achados relacionados à intervenção.

Agradecimentos: ao Conselho Nacional de Desenvolvimento Científico e Tecnológico (CNPq) e à Fundação de Amparo à Pesquisa do Estado de Pernambuco (Facepe) pelo apoio financeiro.

Declaração: os autores declaram não haver conflitos de interesse científico neste estudo.

\section{REFERÊNCIAS}

1. Pedrinelli A, Garcez-Leme LE, Nobre RSA. O efeito da atividade física no aparelho locomotor do idoso. Rev Bras Ortop. 2009;44(2):96-101.

2. Cubas ER, Boeving A, Marcatto C, Santos CMC, Borba VCZ, Culak CAM. Principais causas de diminuição da massa óssea em muIheres na pré-menopausa encaminhadas ao ambulatório de doenças ósteo-metabólicas de um Hospital Terciário de Curitiba. Arq Bras Endocrinol Metab. 2006;50(5):914-9.

3. World Health Organization. Assessment of fracture risk and its role application to screening for postmenopausal osteoporosis. WHOTech Rep Ser. 1994;843:121-9.

4. Bales CW, Ritchie CS. Sarcopenia, weight loss, and nutritional frailty in the elderly. Annu Rev Nutr. 2002;22:309-23.

5. Aiken J, Bua E, Cao Z, Lopez M, Wanagat J, Mackenzie D, et al. Mitochondrial DNA deletion mutations and sarcopenia. Ann NY Acad Sci. 2002;959:412-23.

6. Nóbrega $A C L$, Freitas $E V$, Oliveira $M A B$, Leitão $M B$, Lazzoli JK, Nahas RM, et al. Posicionamento oficial da Sociedade Brasileira de Medicina do Esporte e da Sociedade Brasileira de Geriatria e Gerontologia: atividade física e saúde no idoso. Rev Bras Med Esporte. 1999;5(6):207-11.

7. Ikemoto Y, Demura S, Yamaji S, Minami M, Nakada M, Uchiama M. Force-time parameters during explosive isometric grip correlate with muscle power. Sport Sci Health. 2007;2(2):64-70.

8. Geraldes AAR, Oliveira ARM, Albuquerque RB, Carvalho JM de, Farinatti P de VT. A força de preensão manual é boa preditora do desempenho funcional de idosos frágeis: um estudo correlacional múltiplo. Rev Bras Med Esporte. 2008;14(1):12-6.

9. Grimley-Evans J. Fallers, non-fallers and Poisson. Age Ageing. 1990;19:268-69.

10. Carter ND, Kannus P, Khan KM. Exercise in the prevention of falls in older people: a systematic literature review examining the rationale and the evidence. Sports Med. 2001;31(6):427-38.

11. Gass M, Dawson-Hughes B. Preventing osteoporosis-related fractures: an overview. Am J Med. 2006;119(4):3-11.

12. Borba-Pinheiro CJ, Carvalho MCG de A, Silva NSA da, Bezerra JCP, Drigo AJ, Dantas EHM. Efeitos do treinamento resistido so- bre variáveis relacionadas com baixa densidade óssea de muIheres menopausadas tratadas com alendronato. Rev Bras Med Esporte. 2010;16(2):121-5.

13. Papaioannou A, Adachi JD, Winergard K, Ferko N, Parkinson W, Cook RJ, et al. Efficacy of home-based exercise for improving quality of life among elderly women with symptomatic osteoporosis-related vertebral fractures. Osteoporos Int. 2003;14(8):677-82.

14. Kerr D, Ackland T, Maslen B, Morton A, Prince R. Resistance training over 2 years increase bone mass in calcium-replete postmenopausal women. J Bone Miner Res. 2001;16(1):175-81.

15. Ryan AS, Ivey FM, Hurlbut DE, Martel GF, Lemmer JT, Sorkin $J D$, et al. Regional bone mineral density after resistive training in young and older men and women. Scand J Med Sci Sports. 2004;14(1):16-23.

16. Figliolino JAM, Morais TB, Berbel AM, Corsol SD. Análise da influência do exercício físico em idosos com relação a equilíbrio, marcha e atividade de vida diária. Rev Bras Geriatr Gerontol. 2009;12(2):227-38.

17. Brucki $S M D$, Nitrini $R$, Caramelli $P$, Bertolucci PHF, Okamoto IH. Sugestões para o uso do mini-exame do estado mental no Brasil. Arq Neuropsiquiatr. 2003;6(3-B):777-81.

18. Haidar SG, Kumar D, Bassi RS, Desmukh SC. Average versus maximum grip strength: Which is more consistent? J Hand Surg Br. 2004;29(1):82-4.

19. Dias JA, Ovando AC, Külkamp W, Borges-Júnior NG. Força de preensão palmar: métodos de avaliação e fatores que influenciam a medida. Rev Bras Cineantropom Desempenho Humano. 2010;12(3):209-16.

20. Pfeifer M, Minne HW. Bone loading exercise recommendations for prevention and treatment of osteoporosis. IOF International Osteoporosis Foundation: Committee of Scientific Advisors; 2005.

21. Moser AD de L, Malucelli MF, Bueno SN. Cadeia cinética aberta e fechada: uma reflexão crítica. Fisioter Mov. 2010;23(4):641-50.

22. Sherrington C, Whitney JC, Lord SR, Hebert RD, Cumming RG, Close JCT. Effective exercise for the prevention of falls: a systematic review and meta-analysis. J Am Geriatr Soc. 2008;56(12):2234-43.

23. Sinaki M, Itoi E, Wahner HW, Wollan P, Gelzcer R, Mullan BP, et al. Stronger back muscles reduce the incidence of vertebral fractures: a prospective 10 year follow-up of postmenopausal women. Bone. 2002;6:836-41.

24. Kemmler W, Lauber D, Weineck J, Hensen J, Kalender W, Engelhe $\mathrm{K}$. Benefits of 2 years of intense exercise on bone density, physical fitness, and blood lipids in early postmenopausal osteopenic women results of the Erlangen Fitness Osteoporosis Prevention Study (EFOPS). Arch Intern Med. 2004;164:1084-91.

25. Cussler EC, Going SB, Houtkooper LB, Stanford VA, Blew RM, Flint-Wagner $\mathrm{HG}$, et al. Exercise frequency and calcium intake predict 4-year bone changes in postmenopausal women. Osteoporosis Int. 2005;16:2129-41.

26. Skelton DA, Young A, Greig CA, Malbut KE. Effects of resistance training on strength, power, and selected and functional abilities of women aged 75 and older. J Am Geriatr Soc. 1995;43(10):1081-7.

27. Kisner C, Colby LA. Exercícios terapêuticos: fundamentos e técnicas. 5.ed. São Paulo: Manole; 2009. p. 171-215.

28. Lindsey C, Brownbill RA, Bohannon RA, llich JZ. Association of physical performance measures with bone mineral density in postmenopausal women. Arch Phys Med Rehabil. 2005;86(6):1102-7.

29. Rhodes EC, Martin AD, Tauton JE, Donnelly M, Warren J, Elliot $J$. Effects of one year of resistance training on the relation between muscular strength and bone density in elderly women. $\mathrm{Br} \mathrm{J}$ Sports Med. 2000;34(1):18-22.

30. Marin RV, Pedrosa MAC, Moreira-Pfrimer LDF, Matsudo SMM, Lazaretti-Castro M. Association between lean mass and handgrip strength with bone mineral density in physically active postmenopausal women. J Clin Densitom. 2010;13(1):96-101.

31. Lima RM, Bezerra LMA, Rabelo HT, Silva MAF, Silva AJR, Bottaro $M$, et al. Fat-free mass, strength, and sarcopenia are related to bone mineral density in older women. J Clin Densitom. 2009;12(1):35-41. 